\title{
Electrostatic Storage Ring With Focusing Provided By the Space Charge of an Electron Plasma
}

\author{
J. L. Pacheco, C. A. Ordonez, and D. L. Weathers \\ Department of Physics, University of North Texas, Denton, Texas 76203
}

\begin{abstract}
Electrostatic storage rings are used for a variety of atomic physics studies. An advantage of electrostatic storage rings is that heavy ions can be confined. An electrostatic storage ring that employs the space charge of an electron plasma for focusing is described. An additional advantage of the present concept is that slow ions, or even a stationary ion plasma, can be confined. The concept employs an artificially structured boundary, which is defined at present as one that produces a spatially periodic static field such that the spatial period and range of the field are much smaller than the dimensions of a plasma or charged-particle beam that is confined by the field. An artificially structured boundary is used to confine a non-neutral electron plasma along the storage ring. The electron plasma would be effectively unmagnetized, except near an outer boundary where the confining electromagnetic field would reside. The electron plasma produces a radially inward electric field, which focuses the ion beam. Self-consistently computed radial beam profiles are reported.
\end{abstract}

Keywords: electrostatic storage ring, ion focusing, non-neutral electron plasma

PACS: 29.20.db, 52.20.Hv, 52.27.Jt

\section{INTRODUCTION}

Several purely electrostatic systems can be employed to confine ion beams or drifting ion plasmas, such as electrostatic storage rings, electrostatic ion beam traps, Kingdon traps, and electrostatic beam guides [1-8]. Confinement of charged particles by purely electrostatic means is important when time-varying electromagnetic or magnetostatic confinement interfere with the experiment at hand. A system is proposed here for investigation of atomic physics processes in plasmas (e.g., processes characterized by the interactions of trapped heavy ions and plasma electrons). A system is envisioned where a hollow and evacuated cylinder, which is made from a refractory metal such as tungsten or tantalum, is heated to a temperature sufficient for thermionic electron emission to occur from the interior surface. A non-drifting, non-neutral electron plasma would be produced within the interior of such a cylinder, and an electrostatic potential well for positive particles would be created by the space charge of the electron plasma. Similarly, suppose that a cylindrical beam line has an interior surface lined with an artificially structured boundary [9] so that it confines an electron plasma. Under certain conditions, the space charge of that electron plasma can be used to focus a positive-ion beam or drifting plasma; see

Application of Accelerators in Research and Industry

AIP Conf. Proc. 1525, 88-93 (2013); doi: 10.1063/1.4802296

(C) 2013 AIP Publishing LLC 978-0-7354-1148-7/\$30.00
Fig.1. Note that focusing is loosely defined here as the nominal effect of ion bunching towards a desired region, and its definition is not the typical ion optics one. In the work presented here, the equilibrium of a two-species system, with one species confined by the space charge of the other, is self-consistently evaluated. Each species is assumed to be radially relaxed to a Boltzmann density distribution. An edgeconfined electron plasma would be effectively unmagnetized except near an outer boundary where a confining electromagnetic field would reside. One possibility is for the confining electromagnetic field to consist of a spatially periodic sequence of magnetic cusps that are plugged electrostatically. This is a case where a magnetic multipole would be superimposed on an electric multipole of higher order. The spatial period and range of the field would be much smaller than the dimensions of the plasma, such that the positively charged species (beam or plasma) would experience only an electrostatic field that is produced by the space charge of the electron plasma. 

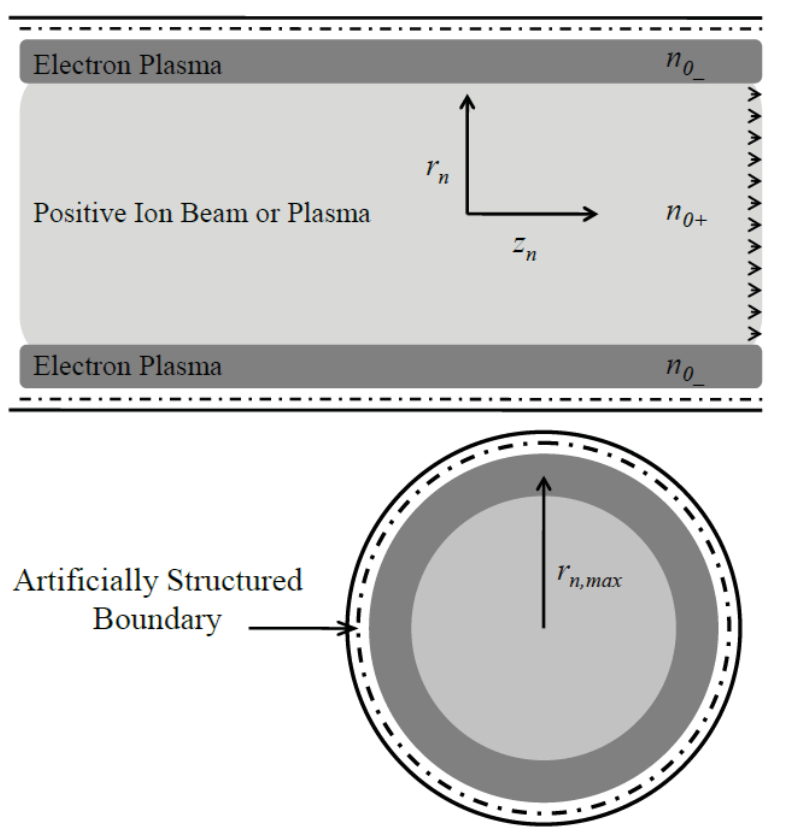

FIGURE 1. Cross-sections of a segment of a cylindrical beam line. The electron plasma is confined by an artificially structured boundary. The space charge of the electron plasma creates an electrostatic potential that focuses a positive-ion beam or drifting plasma.

\section{THEORY}

Consider a surface-emitted or edge-confined electron plasma that follows a Boltzmann distribution in the radial direction of an infinitely long, cylindrically symmetric device. Such a configuration serves as a model for a storage ring that has a ring radius that is much larger than the characteristic electron plasma parameters. An electrostatic potential well is therefore created at the radial center of such a device for positive particles. Assume that a positively charged species, such as a positive-ion or positron beam or plasma, is introduced near the center of the electron plasma. The charge density is $\rho(r)=$ $-e n_{-}(r)+Z e n_{+}(r)$, where $Z$ represents the average charge state of the positive species. The first term on the right-hand side refers to the electron plasma, which follows a Boltzmann density profile of the form $n_{-}(r)=n_{0_{-}} e^{\frac{e\left[\varphi(r)-\varphi\left(r_{s}\right)\right]}{T_{-}}}$. The second term refers to the positive particle beam or plasma that is confined by the electrostatic potential well created by the space charge of the electron plasma. The positive species distribution is also assumed to be of a Boltzmann type. Its density profile is $n_{+}(r)=n_{0_{+}} e^{\frac{-Z e[\varphi(r)-\varphi(0)]}{T_{+}}}$. From this point on, we refer to a positive ion beam or plasma as positive species where the temperature $T_{+}$is associated with the transverse energy of the positive ion beam, or $T_{+}$is the characteristic temperature of the positive species when it is referred to as a plasma.

Poisson's equation is

$$
\begin{aligned}
& \nabla^{2} \varphi(r)= \\
& \quad \frac{-1}{\varepsilon_{0}}\left(-e n_{0_{-}} e^{\frac{e\left[\varphi(r)-\varphi\left(r_{S}\right)\right]}{T_{-}}}+Z e n_{0_{+}} e^{\frac{-Z e[\varphi(r)-\varphi(0)]}{T_{+}}}\right),
\end{aligned}
$$

where $n_{-}\left(r_{s}\right)=n_{0_{-}}$and $n_{+}(0)=n_{0_{+}}$are known densities of the respective species. Let $N_{n}=Z \frac{n_{0_{+}}}{n_{0_{-}}}$and $T_{n}=Z \frac{T_{-}}{T_{+}} . N_{n}$ is the positive species charge density at the center of the configuration normalized by the magnitude of the electron charge density at the edge. $T_{n}$ is the average charge state of the positive species multiplied by the ratio of the electron temperature to the temperature of the positive species. Equation (1) can then be written as

$$
\begin{aligned}
& \nabla^{2} \varphi(r)= \\
& \quad \frac{-e n_{0-}}{\varepsilon_{0}}\left(-e^{\frac{e\left[\varphi(r)-\varphi\left(r_{S}\right)\right]}{T_{-}}}+N_{n} e^{\frac{-e T_{n}[\varphi(r)-\varphi(0)]}{T_{-}}}\right)
\end{aligned}
$$

Introducing the normalized electrostatic potential, $\psi(r)=\frac{e}{T_{-}} \varphi(r)$ with $\varphi\left(r_{s}\right)=0$, where $r_{s}$ is the boundary of the system gives

$$
\nabla^{2} \psi(r)=\frac{-e^{2} n_{0-}}{\varepsilon_{0} T_{-}}\left(-e^{\psi(r)}+N_{n} e^{-T_{n}[\psi(r)-\psi(0)]}\right) .
$$

Then defining the Debye length at the boundary of the electron plasma as $\lambda_{D_{-}}^{2}=\frac{\varepsilon_{0} T_{-}}{e^{2} n_{0_{-}}}$and scaling the coordinates with respect to this quantity produces

$$
\nabla^{2} \psi\left(r_{n}\right)=e^{\psi\left(r_{n}\right)}-N_{n} e^{-T_{n}\left[\psi\left(r_{n}\right)-\psi(0)\right]},
$$

where $r_{n}=\frac{r}{\lambda_{D_{-}}}$is the normalized radial coordinate, and $\psi(0)$ is the value of the normalized electrostatic potential at the center of the system. For a storage ring, in the limit of a large ring radius, a cylindrical geometry is assumed. The Laplacian operator in Eq. (4) takes its usual cylindrical form. Note that the system has been scaled with respect to the electron plasma Debye length. However, quantities pertaining to the positive species can be obtained via the following relationships:

$$
\lambda_{D_{-}}^{2}=N_{n} T_{n} \frac{\varepsilon_{0} T_{+}}{e^{2} Z^{2} n_{0_{+}}}=N_{n} T_{n} \lambda_{D_{+}}^{2}
$$


and

$$
\psi_{+}\left(r_{n}\right)=-T_{n} \psi\left(r_{n}\right)
$$

The notation $\psi\left(r_{n}\right)=\psi_{-}\left(r_{n}\right)$ could be used in Eqs. (3), (4), and (5), and note that electron and positive species Debye lengths are specified with respect to the densities at the boundary and the center of the system, respectively.
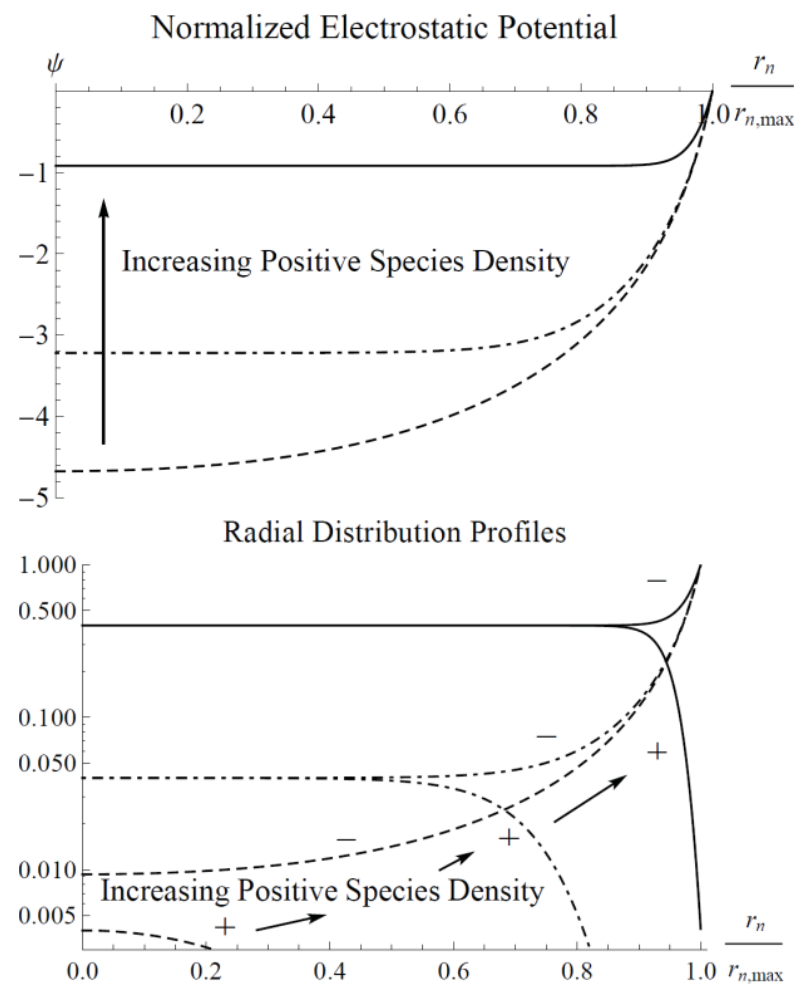

FIGURE 2. Normalized electrostatic potential of a twospecies system (top). Self-consistent distributions of the two species (bottom) in logarithmic scale. The plots are for $T_{n}=$ $5, r_{n, \max }=30$, and $N_{n}=0.004$ (dashed), 0.04 (dot-dashed), 0.4 (solid). The arrows indicate the trend that the system follows as $N_{n}$ is increased. In the lower panel of this figure and Figs. 3, 4, and 5, the normalized distributions are $n_{-}\left(r_{n}\right) / n_{0_{-}}$, which are labeled by minus signs $(-)$, and $Z n_{+}\left(r_{n}\right) / n_{0_{-}}$, which are labeled by plus signs $(+)$. Thus, each matching pair of plots are the normalized distributions for the negative and positive species. are:

The boundary conditions for the two-species system

Neumann Boundary Condition

$$
\left[\frac{\partial \varphi(r)}{\partial r}\right]_{r=0}=\left[\frac{\partial \psi\left(r_{n}\right)}{\partial r_{n}}\right]_{r_{n}=0}=0, \text { and }
$$

Dirichlet Boundary Condition

$$
\varphi\left(r_{\max }\right)=\psi\left(r_{n, \max }\right)=0
$$

where $r_{\max }=r_{s}$ and $r_{n, \max }=\frac{r_{s}}{\lambda_{D_{-}}}$.

A finite-differences approach was used to selfconsistently solve Eq. (4) to calculate the properties of the system when the two species are present [10]. The number of grid points was determined by the smallest Debye length (smallest of the two species). At least three grid points per such Debye length were used. The electrostatic potential was iteratively calculated in a self-consistent manner until the absolute difference between one iteration and the next was less than $10^{-10}$ at all grid points.

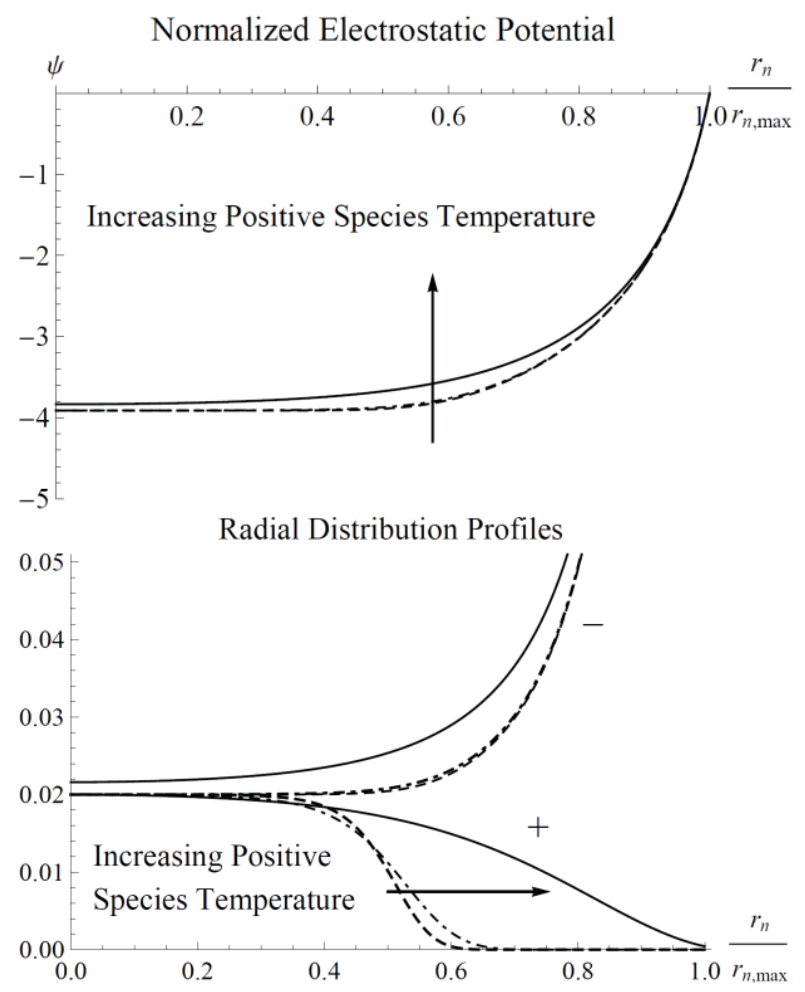

FIGURE 3. Normalized electrostatic potential of a twospecies system (top). Self-consistent distributions of the two species (bottom). The plots are for $N_{n}=0.02, r_{n, \max }=30$, and $T_{n}=1$ (solid), 15 (dot-dashed), and 30 (dashed). The \pm labels are defined in Fig. 2.

\section{RESULTS}

For sufficiently high densities of the positive species, both species approach the same charge density at the center of the system, and the electrostatic potential well depth decreases as the charge density of the positive species is increased. Such behavior is shown in Fig. 2. If the positive species charge density is sufficiently small, the electrostatic potential 
generated by, and density of, the electron plasma are unaffected by the presence of the positive species. However, Fig. 3 shows that as the temperature of the positive species is increased, its distribution occupies a larger volume. The normalized electrostatic potential and distribution profiles for two species with equal temperatures and charge states under equilibrium conditions are now examined. Figure 4 shows a twospecies system in which these have equal temperatures and charge states. Notice that for sufficiently high charge densities of the positive species, the twospecies system reaches neutrality near the center. When the charge density of the positive species is lower, there exists a state of partial neutralization.
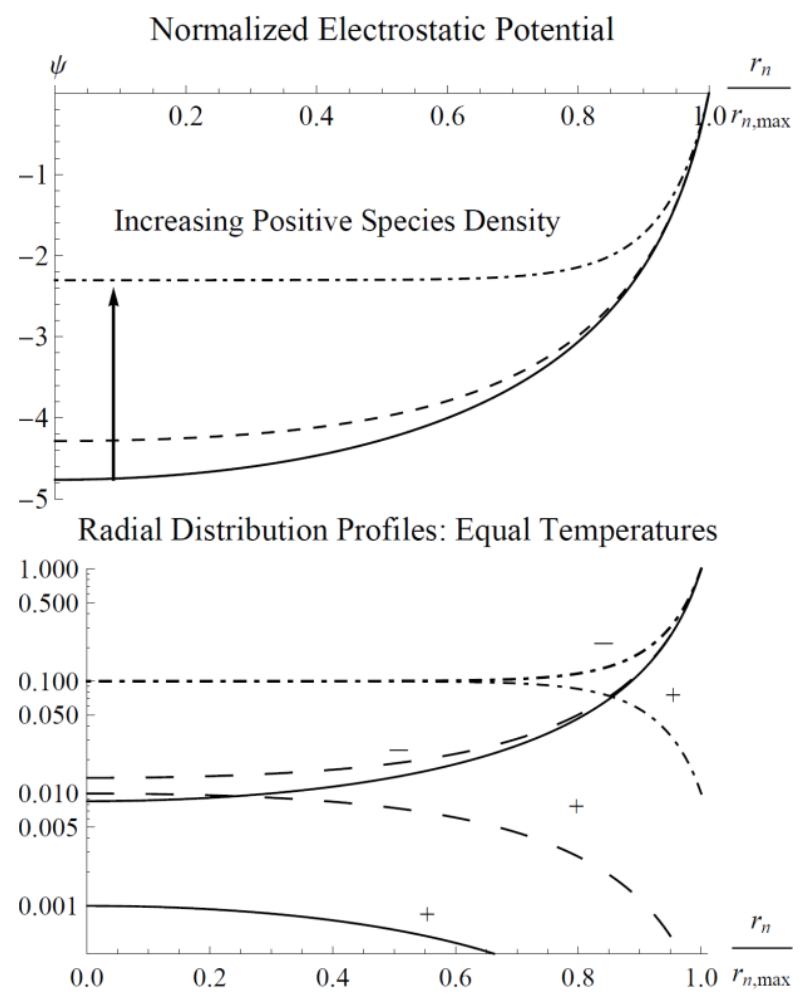

FIGURE 4. Two-species system with equal temperatures and charge states. The plots are for $T_{n}=1, r_{n, \max }=30$, and $N_{n}=0.1$ (dot-dashed), 0.01 (dashed), and 0.001 (solid). The \pm labels are defined in Fig. 2.

As shown in Fig. 4, relatively high (low) densities of the positive species can yield a region of neutrality (partial neutralization) near the center of the twospecies system. Figure 5 shows the minimum positive species charge density at which the two-species system achieves approximate charge neutrality at the center for different temperatures or charge states of the two species. The minimum neutral density decreases as the positive species temperature is lowered or its average charge state is increased.
The space charge of the electron plasma becomes partially compensated as the charge density of the positive species is increased at the center of the configuration. Consequently, the electrons near the center of the configuration experience less repulsion from each other (due to the presence of the positive species), and the two-species system self-consistently adjusts to an equilibrium in which the central electron density is higher than would occur if the positive species were absent.
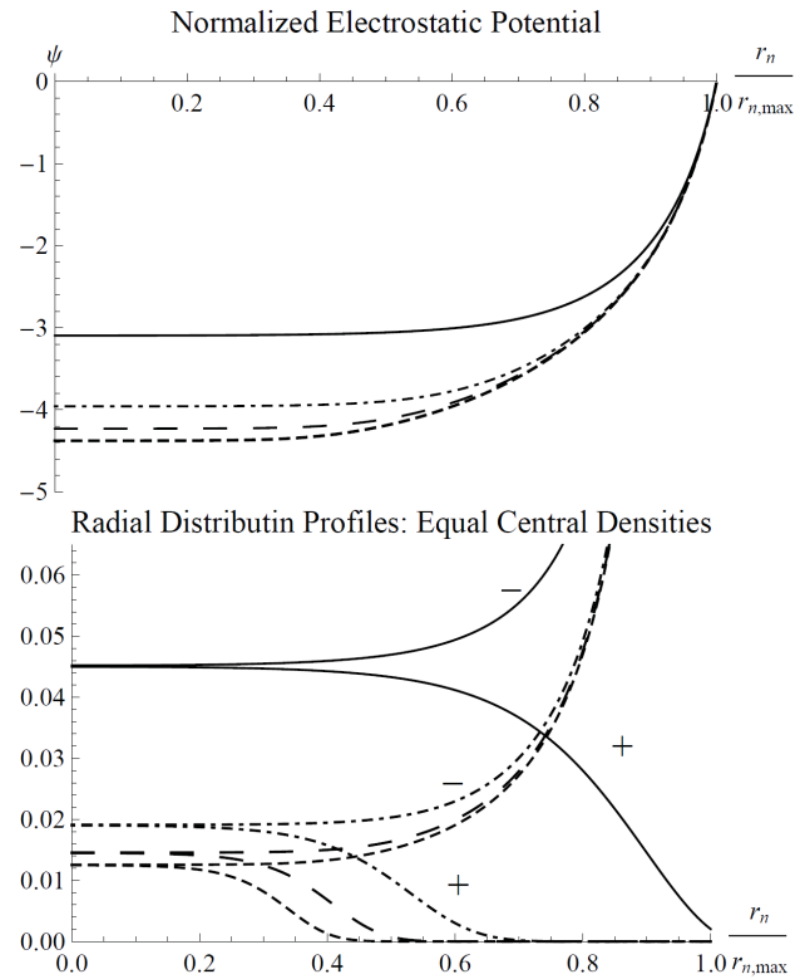

FIGURE 5. Two-species system with approximately equal charge densities at the center. The plots are for $r_{n, \max }=30$ and $\left(T_{n}, N_{n}\right)=(1,0.05)$ [solid], $(10,0.0225)$ [dot-dashed], $(25,0.0152)$ [long dash], and $(40,0.0145)$ [short dash]. The normalized electron temperatures $T_{n}$ were chosen and the normalized positive species charge densities $N_{n}$ were then adjusted to the lowest value at which the two distributions have approximately the same value at the center of the system. The \pm labels are defined in Fig. 2.

\section{SPACE-CHARGE-BASED ELECTROSTATIC FOCUSING}

Focusing of the positive species is expected to occur when the electrostatic potential energy well created by the electron plasma is much deeper than the kinetic energy associated with the transverse temperature of the positive species [11]: 


$$
T_{+} \ll Z e \Delta \varphi_{0} .
$$

Here, $\Delta \varphi_{0}=\varphi\left(r_{\max }\right)-\varphi(0)$ is the ordinary (unnormalized) electrostatic potential difference between the edge and the center of the configuration. In terms of a normalized quantity, the condition is written as

$$
\Delta \psi_{+0}=\frac{Z e \Delta \varphi_{0}}{T_{+}} \gg 1
$$

$\Delta \psi_{+0}$ is the normalized electrostatic potential energy well depth that confines the positive species. Also, $\Delta \psi_{0}$ is the electrostatic potential energy well created by the electron plasma; that is,

$$
\Delta \psi_{0}=\frac{\Delta \psi_{+0}}{T_{n}} .
$$

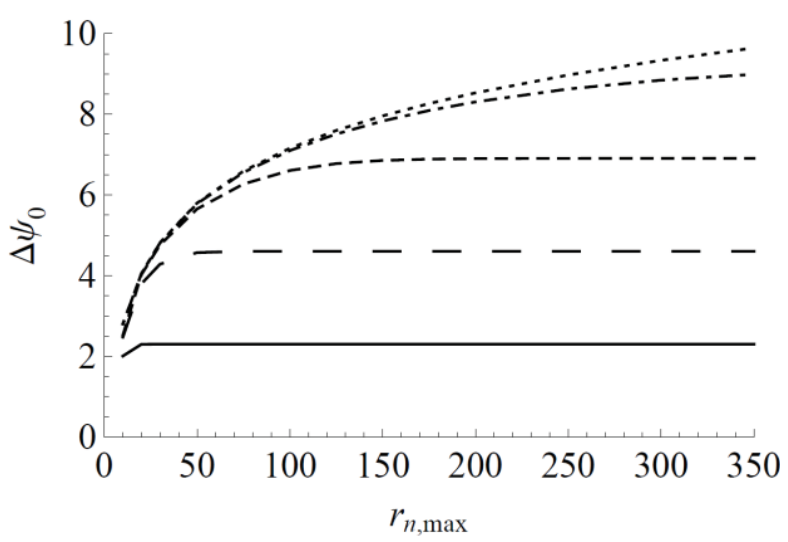

FIGURE 6. Normalized electrostatic potential energy well depth for space-charge-based electrostatic focusing as a function of normalized system size. The plots are for $T_{n}=1$ and $N_{n}=0$ (dotted), 0.0001 (dot-dashed), $0.001 \quad$ (dash), 0.01 (long dash), and 0.1(solid).

$\Delta \psi_{0}$ is evaluated in Fig. 6. To explore the effect that $N_{n}$ has on $\Delta \psi_{0}$, this quantity was evaluated as a function of system size for a range of values. It is found that the normalized electrostatic potential energy well depth tends to decrease as the normalized charge density of the positive species is increased and to increase with normalized system size. However, for a sufficiently large normalized size, the normalized well depth saturates to a value nearly independent of the normalized size of the system. The electrostatic potential energy well depth experienced by the positive species also increases with the temperature ratio according to Eq. (10). Figure 7 shows $\Delta \psi_{0}$, the normalized electrostatic potential difference, evaluated for several values of $T_{n}$ and $N_{n}$. Assuming that a state of charge neutrality exists at the center of the system, the normalized electrostatic potential difference is given by the following expression for $r_{n, \max } \gtrsim$ 200 and $10^{-4} \lesssim N_{n} \leq 1$ :

$$
\frac{\Delta \psi_{+0}}{T_{n}}=\Delta \psi_{0} \approx-\ln \left(N_{n}\right)
$$

This result can be used together with collision-based theory, such as that in [11] and [12], to evaluate spacecharge-based electrostatic confinement time scales.

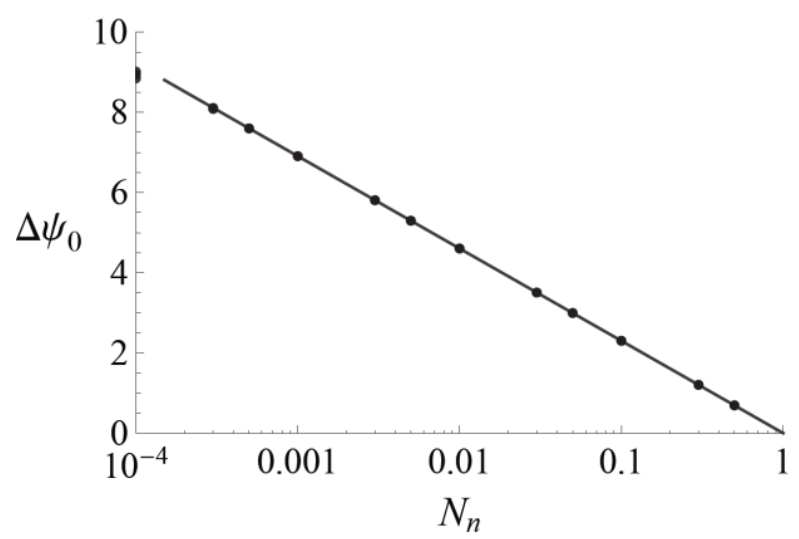

FIGURE 7. Normalized electrostatic potential difference for increasing normalized charge density of the positive species. Points are plotted for $r_{n, \max }=300$ and $T_{n}=1,4$, and 10 for each value of $N_{n}$, but these are indistinguishable for the different values of $T_{n}$. The solid line drawn through the points is from Eq. (11).

\section{CONCLUSION}

A self-consistent computation of the electrostatic potential generated by the space charge of an edgeconfined or surface-emitted electron plasma that follows a Boltzmann density distribution in the radial direction of a cylindrically symmetric system has been carried out. The electrostatic potential energy well generated was employed to investigate the possible focusing of a positive-ion beam or drifting plasma. The results obtained are applicable for a cylindricallysymmetric charged-particle focusing system such as a beam guide or a storage ring in the limit of large ring radius. The two-species system has been characterized by varying normalized parameters, which include (1) the ratio of the plasma radius to the Debye length at the plasma edge, (2) the ratio of the positive species charge density at the center of the system to that of the electron plasma at the edge, and (3) the ratio of the temperatures multiplied by the average charge state.

The electrostatic potential profile and the charge density distribution have been computed, and the cases explored indicate the following: (1) increasing the charge density of the positive species decreases the 
depth of the electrostatic potential well; (2) increasing the temperature or decreasing the average charge state of the positive species causes the positive species to occupy a larger volume; (3) an equilibrium is possible in which the two species have equal temperatures and equal charges states; and (4) approximately equal charge densities of the two species at the center of the system occur for a sufficiently high charge density of the positive species, even when the temperatures and charge states are equal.

The electrostatic potential well depth has been evaluated for the two-species system. It has been found that once the positive species is introduced, the electrostatic potential well depth reaches a saturation regime when the normalized size is sufficiently large. The electrostatic potential well depth has been described analytically in this saturated regime. The results obtained can be applied to evaluate spacecharge-based electrostatic confinement time scales. The concept could be of particular utility for investigation of atomic or molecular processes in plasmas.

\section{ACKNOWLEDGEMENTS}

This material is based upon work supported by the Department of Energy under Grant No. DE-FG0206ER54883 and by the National Science Foundation under Grant No. PHY-1202428.

\section{REFERENCES}

1. J. U. Andersen, P. Hvelplund, S. B. Nielsen, S. Tomita, H. Wahlgreen, S. P. Møller, U. V. Pedersen, J. S. Forster, and T. J. D. Jørgensen, Rev. Sci. Instrum. 73, 1284 (2002).

2. T. Tanabe, K. Chida, K. Noda, and I. Watanabe, Nucl. Instrum. Methods Phys. Res. A 482, 595 (2002).

3. D. Zajfman, O. Heber, L. Vejby-Christensen, I. BenItzhak, M. Rappaport, R. Fishman, and M. Dahan, Phys. Rev. A 55, R1577 (1997).

4. M. Dahan, R. Fishman, O. Heber, M. Rappaport, N. Altstein, D. Zajfman, and W. J. van der Zande, Rev. Sci. Instrum. 69, 76 (1998).

5. H. T. Schmidt, H. Cederquist, J. Jensen, and A. Fardi, Nucl. Instrum. Methods Phys. Res. B 173, 523 (2001).

6. Y. Ishida, M. Wada, and H. Wollnik, Nucl. Instrum. Methods Phys. Res. B 241, 983 (2005).

7. C. A. Ordonez, Phys. Plasmas 15, 072508 (2008).

8. C. A. Ordonez, J. Appl. Phys. 104, 054903 (2008).

9. C.A. Ordonez. J. Appl. Phys. 106, 024905 (2009).

10. W. H. Press, S. A. Teukolsky, W. T. Vetterling, and B. P. Flannery, Numerical Recipes: The Art of Scientific Computing. Cambridge University Press, Cambridge, 2007.

11. Y. Chang, J. R. Correa, and C. A. Ordonez, Phys. Plasmas 11, 3360 (2004).
12. J. R. Correa, Y. Chang, and C. A. Ordonez, Phys. Plasmas 12, 084505 (2005). 